\title{
ACCURACY OF SPINDLE UNITS WITH HYDROSTATIC BEARINGS
}

\author{
Dmytro FEDORYNENKO*, Serhii SAPON*, Sergiy BOYKO*
}

\author{
*Chernihiv National University of Technology, Mechanical Engineering Department, Shevchenko Street, 95, Chernihiv, Ukraine
}

fdy1974@gmail.com, s.sapon@gmail.com, svboyko.cstu@gmail.com

received 2 March 2015, revised 11 May 2016, accepted 13 May 2016

\begin{abstract}
The work is devoted to the research ofprecision regularities in a spindle unit by the trajectory of the spindle installed on hydrostatic bearings. The mathematical model of trajectories spindle with lumped parameters that allows to define the position of the spindle with regard the simultaneous influence of design parameters, geometrical deviations ofform, temperature deformation bearing surfaces, the random nature of operational parameters and technical loads of hydrostatic bearings has been developed. Based on the results of numerical modeling the influence of shape errors of bearing surface of hydrostatic bearing on the statistical characteristics of the radius vector trajectories of the spindle by varying the values rotational speed of the spindle and oil pressure in front hydrostatic bearing has been developed. The obtained statistical regularities of precision spindle unit have been confirmed experimentally. It has been shown that an effective way to increase the precision of spindle units is to regulate the size of the gap in hydrostatic spindle bearings. The new design of an adjustable hydrostatic bearing, which can improve the accuracy of regulation size gap has been proposed.
\end{abstract}

Key words: Spindle, Hydrostatic Bearing, Spindle Unit, Adjustable Hydrostatic Bearing, Machining Accuracy, Trajectory, Bearing Surface Imperfection

\section{INTRODUCTION}

One of the most important formative units of any machine tool is the spindle unit (SU) the output accuracy parameters of which are strongly dependent on the type of spindle bearings. An efficient way to increase the accuracy of machine tools is to use as spindle bearings hydrostatic bearings $(\mathrm{HB})$ adjustable type. It will provide the expansion of technological capabilities of machine tools by combining roughing and finishing, the increase of efficiency of processing based on high speed bearings and structural designs and reducing operating costs by adjusting geometric and operating parameters of $\mathrm{HB}$ depending on the nature of technological load (Savin, 2006; Shen et al., 2010; Solomin, 2007; Wardle, 2015; Perovic, 2012).

The main parameter of the precision of a SU is the trajectory of spatial movement of fixed points located on the auxiliary spindle bases that determine the position of the cutting tool or the device with fixed workpiece (Fedorynenko et al., 2015; Savin, 2006; Strutynsky and Fedorynenko, 2011). The trajectory of the spindle motion is formed under the influence of totality variables in magnitude and the direction of the external and internal efforts with determined and stochastic components: cutting force, reaction in $\mathrm{HB}$, the efforts from the main motion, static and dynamic unbalance from rotating of workpiece in the spindle, technological equipment, which is fixed in (on) spindles, etc. The analysis of regularities of formation of spindle trajectories under such system of forces with taking into account the shape errors at the same time, elastic and temperature deformations of bearing surfaces is an actual scientific problem. Solving of this problem will determine rational design and operating parameters of spindle units, which will improve the accuracy of machine tools and expand their technological capabilities.

\section{MATHEMATICAL MODEL TRAJECTORIES OF SPINDLE WITH HYDROSTATIC BEARINGS}

The basic method for the theoretical analysis of the spatial movement of the spindle with $\mathrm{HB}$ is a method of trajectories (Fedorynenko et al., 2015; Xiaodong and Huaimin, 2006; Xiaodong et al., 2007), based on the numerical integration of the system equations of hydrodynamics lubricant layer and the equations of the spindle motion to simulate a real dynamic behavior of the spindle, considering the influence of nonlinear reactions of lubricant layer, operating parameters of $\mathrm{HB}$, technological power loads etc.

The object of the modeling is a SU of the precision lathe UT16A (Fig. 1), which spindle 3 is mounted on two radial HB 1 and 6 and thrust bearing, formed between the ends of bushings 5 and 7 and housing of front radial $\mathrm{HB}$.

Spindle position in the space under loading force and reactions in HB is described by the trajectory of its axis. Taking into account that the axial displacement of the spindle lathe does not affect the accuracy of forming a radial direction, the study a spindle precision unit has been carried out by its performance trajectories of motion in the plane perpendicular to the geometrical axis.

The offset of the spindle under the power loads and reactions of lubricant layer are represented as an equivalent single-mass dynamic model (Fig. 2), which includes the resulted mass $m$, which is displaced in the vertical $\Delta y$ and horizontal $\Delta x$ directions under force loads and resultant reactions in HB reduced to the cutting area projections. As a result of a limited impact of characteristics of rear $\mathrm{HB}$ on machining precision, the reactions of a lubricant layer, that arise in rear HB were not considered. 


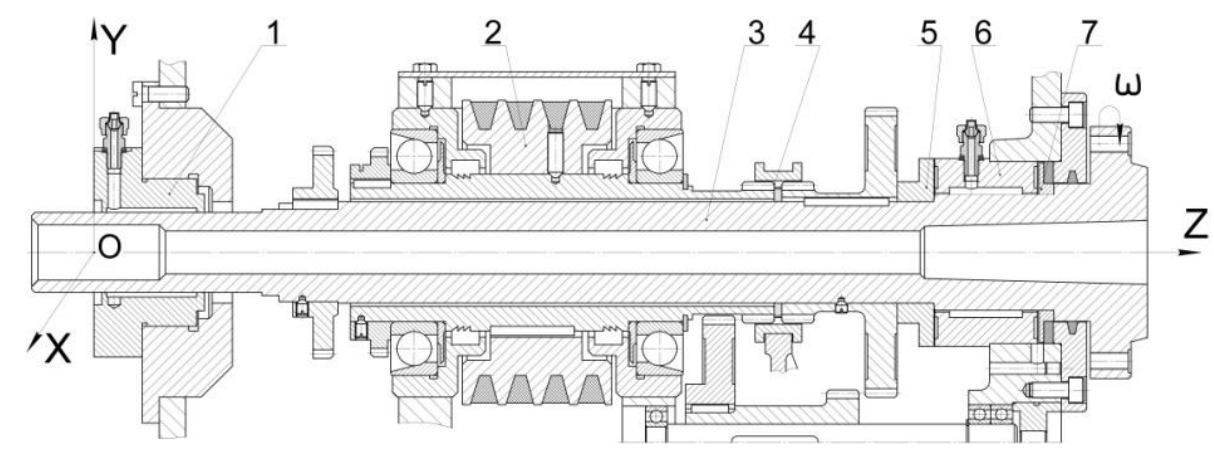

Fig. 1. Constructive scheme of spindle lathe UT16A: 1, 6 - radial HB; 2 - pulley drive; 3 - spindle; 4 - gear coupling; 5, 7 - bushings of axial HB

In the drive of main machine tool UT16A motion belt transmission unloaded from efforts tension of belts, is used and efforts of the drive are represented by a random component, which is caused by shock processes in gears in gear couplings 4 (see Fig. 1).

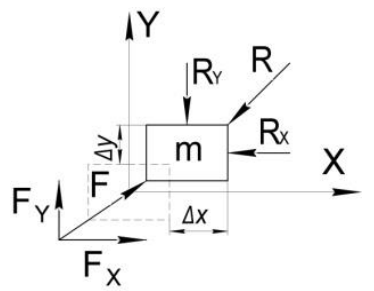

Fig. 2. The equivalent single-mass dynamic model of the spindle

Taking into account all previously made assumptions the system of differential equations that describe the displacement of the spindle in the plane perpendicular to its geometrical axis will look like:

$\left\{\begin{array}{l}m \frac{d^{2} x}{d \tau^{2}}=F_{x}-R_{x} \\ m \frac{d^{2} y}{d \tau^{2}}=F_{y}-R_{y}\end{array}\right.$

where: $m$ - bringing mass of the spindle; $F_{x}, F_{y}, R_{x}, R_{y}-$ projection on the axis $O X$ and $O Y$ respectively resultant power loads on the spindle and the resultant reactions in $\mathrm{HB}$.

Projections $F_{x}, F_{y}$ resultant power loads on the spindle, bringing to the cutting area, are defined as follows:

$F_{x}=\chi_{c} F_{C x}+\chi_{R} F_{p r x}+\chi_{P} P_{x}$

$F_{y}=\chi_{c} F_{C y}+\chi_{R} F_{p r y}+\chi_{P} P_{y}+\chi_{G} m g$

where: $\chi_{C}, \chi_{R}, \chi_{P}, \chi_{G}$ - coefficients of bringing forces and the mass to the cutting area; $F_{C x}, F_{C y}, P_{x}, P_{y}, F_{p r y}, F_{p r x}$ - projections on the axis $O X$ and $O Y$ respectively centrifugal force of the inertia, constituting cutting force and effort on the part of the main drive motion.

It should be noted that virtually all components of resultant power loads (formula (2) and (3)) are random to determine them generally the dependency was used:

$f(\tau)=\bar{m}_{f}+\Delta f \cdot \xi(\tau)$

where: $\bar{m}_{f}, \Delta f$ - mathematical expectation and a range of deviation of its mean value; $\xi(\tau)$ - positioning centered random process of magnitude fluctuation.
Thus, when modeling a random process of fluctuations of angular velocity, its absolute value at a certain time $\tau$ was defined:

$\omega(\tau)=\omega_{n}+\Delta \omega_{n} \cdot q(\tau)$

where: $\omega_{n}, \Delta \omega_{n}-$ nominal values and deviation range of the angular velocity; $q(\tau)$ - random process fluctuations of the angular velocity.

Ranges of the angular velocity were determined on the basis of a priori information about the statistical characteristics of the random change of the spindle angular velocity, which was obtained by a series of additional experiments.

Projections of inertia centrifugal force caused by static unbalance of rotating elements spindle unit, taking into account fluctuations of the angular velocity value were determined by the relationships:

$F_{C x}(\tau)=m \cdot \Delta \cdot\left(\omega_{n}+\Delta \omega_{n} \cdot q(\tau)\right)^{2} \cos \left(\omega_{n} \tau+\varepsilon\right)$,

$F_{C y}(\tau)=m \cdot \Delta \cdot\left(\omega_{n}+\Delta \omega_{n} \cdot q(\tau)\right)^{2} \sin \left(\omega_{n} \tau+\varepsilon\right)$

where: $\Delta-$ the displacement of the mass center caused by static unbalance; $\varepsilon$ - the initial phase of static unbalance.

In the presence of static unbalance of rotating elements of a SU absolute value of the depth of cut at time $\tau$ was defined:

$t(\tau)=t_{n}+\Delta \cdot \cos \left(\omega_{n} \tau+\varepsilon\right)+\Delta t_{n} \cdot \xi(\tau)$

where: $t_{n}$ - nominal depth of cut; $\Delta t_{n}$ - magnitude of the changes allowance; $\xi(\tau)$ - random process fluctuations of cut depth.

Random fluctuations in the process of cutting depth can be realized in the form of "white noise", similar to the angular speed of the spindle rotation, or a harmonic function with random amplitude and initial phase (Rubinstein, 2007; Sapon, 2013):

$\xi(\tau)=a(\tau) \cos \left(\omega_{n} \tau+\alpha_{n}\right)$

where: $a(\tau)$ - amplitude coefficient which takes random values from the interval $[-1 ; 1]$ with the relevant distribution law; $\alpha_{n}-$ random phase of harmonic function with uniform distribution in the interval $[0 ; 2 \pi]$.

Instant value of approach $S_{0}(\tau)$ depend on the nominal value of $S_{0}$, as well as a random variable that takes into account the uneven movement of slide as a result of kinematic errors of the drive navigating shaft:

$S_{0}(\tau)=S_{0}+\Delta S_{0} \cdot \eta(\tau)$

where: $S_{0}, \Delta S_{0}$ - nominal deviation and range of approach; $\eta(\tau)$ - random process fluctuations of approach.

Taking into account the random component of the depth 
of cut, feed, speed spindle, the absolute values of the projections $\mathrm{P}_{x}, \mathrm{P}_{y}$ cutting force at a time $\tau$ were determined by the dependencies:

$$
\begin{aligned}
& \mathrm{P}_{x}(\tau)=10 C_{P x}\left(t_{n}+\Delta \cdot \cos \left(\omega_{n} \tau+\varepsilon\right)+\Delta t_{n} \cdot \xi(\tau)\right)^{x} \\
& \times\left(S_{0}+\Delta S_{0} \cdot \eta(\tau)\right)^{y}\left(\frac{3 D \cdot\left(\omega_{n}+\Delta \omega_{n} q(\tau)\right)}{100}\right)^{n} \cdot K_{P x}(\tau), \\
& \mathrm{P}_{y}(\tau)=10 C_{P y}\left(t_{n}+\Delta \cdot \sin \left(\omega_{n} \tau+\varepsilon\right)+\Delta t_{n} \cdot \xi(\tau)\right)^{x} \\
& \times\left(S_{0}+\Delta S_{0} \cdot \eta(\tau)\right)^{y} \times\left(\frac{3 D \cdot\left(\omega_{n}+\Delta \omega_{n} q(\tau)\right)}{100}\right)^{n} \cdot K_{P y}(\tau)
\end{aligned}
$$

where: $C_{P x}, C_{P y}, n, x, y$-coefficients and exponents, which take into account the type of processing, physical and mechanical properties of the workpiece material and tool, $D$ - diameter of workpiece, $\mathrm{mm} ; K_{P x}(\tau), K_{P y}(\tau)$ - correction factors which take into account the actual processing conditions.

Correction factors $K_{P x}, K_{P y}$, were defined as the product of the instantaneous values of coefficients that take into account the influence of the parameters of the cutting tool and the physical and mechanical properties of the material.

Effort which is caused by shock loads in tooth gearings drive the main spindle movement, is presented in the form of "white noise" the model of which is formed like a random sequence of delta impulse $\delta(\tau i)$ with random amplitude values of $a(\tau)$ :

$F_{p r x}^{c}(\tau)=a(\tau) \sin \alpha \cdot \delta\left(\tau-\tau_{i}\right)$,

$F_{\text {pry }}^{c}(\tau)=a(\tau) \cos \alpha \cdot \delta\left(\tau-\tau_{i}\right)$,

where: $a$ - angle of applying shock pulse; $\tau_{i}$ - random time of occurrence disturbance;

$\delta\left(\tau-\tau_{i}\right)= \begin{cases}1, & \text { if } \tau=\tau_{i} \\ 0, & \text { if } \tau \neq \tau_{i}\end{cases}$

The main influence on parameters of machining precision have the parametrs of front spindle HB. Ignoring the part of reactions, which is caused by friction in the lubricant layer, projection resultant reactions of lubricant layer in front $\mathrm{HB}$ in function of the polar angle $\varphi$ is presented by a part of reactions caused by hydrostatic pressure grease on the bearing neck of the spindle:

$R_{x}=\frac{D_{0}}{2} \int_{0}^{2 \pi} p(\varphi) \cos \varphi d \varphi$,

$R_{y}=\frac{D_{0}}{2} \int_{0}^{2 \pi} p(\varphi) \sin \varphi d \varphi$,

where: $D_{0}$ - diameter of the supporting part in $\mathrm{HB} ; p(\varphi)$ - distribution function of the pressure on the supporting part of $\mathrm{HB}$.

The distribution function of pressure $p(\varphi)$ on the supporting part of regulated HB is determined by double integration of the Reynolds equation and depends in the dynamic radial clearance $p(\varphi)$, which at simultaneous taking into account the errors of form and displacement of support surfaces due to their elastic deformation and temperature, is determined by the dependencies:

$$
\begin{aligned}
& h(\phi)=\delta_{0}-m\left(\Delta r_{\mathrm{T}}^{b}\right)-m\left(\Delta r_{\mathrm{T}}^{S}\right)+\frac{T_{r}^{b}}{2}+\frac{\mathrm{T}_{r}^{S}}{2}+ \\
& +\sum_{k=1}^{N} A_{k}^{b} \sin \left(k \varphi+\psi_{0 k}^{b}\right)-\sum_{k=1}^{Q} A_{k}^{S} \sin \left(k \varphi+\chi_{0 k}^{s}\right)
\end{aligned},
$$

where: $m\left(\Delta r_{\mathrm{T}}^{b}\right), m\left(\Delta r_{\mathrm{T}}^{s}\right)$ - mathematical expectation displacement of radial coordinate profile hydrostatic support surfaces bushings and the spindle caused by temperature deformation in a particular temperature; $\delta_{0}$ - static radial clearance in the support; $T_{r}^{b}, \mathrm{~T}_{r}^{S}$ - roundness tolerances of support surfaces in the bearing; $A_{k}^{b}, \quad \psi_{0 k}^{b}, A_{k}^{s}, \quad \chi_{0 k}^{s}$ - amplitude and initial phase of harmonic
Fourier series which describe the deviation hydrostatic support surfaces bushings and the spindle respectively; $N$ and $Q-$ the number of important harmonics profile of hydrostatic support surfaces bushings and the spindle at a certain temperature.

Details question of the function of the pressure distribution $p(\varphi)$ considering non-ideal geometry and thermal deformation of the bearing support surfaces are considered in the monograph (Fedorynenko et al., 2014; Junpeng et al., 2008; Strutynsky and Fedorynenko, 2011; Yu and Zhang, 2008; Zhao et al., 2007).

Mathematical expectations of displacement of radial coordinate $m\left(\Delta r_{\mathrm{T}}^{b}\right)$ and profile $m\left(\Delta r_{\mathrm{T}}^{s}\right)$ of support surfaces were determined by the results of numerical modeling tools of CAEsystem SolidWorks Simulation (Fig. 3) (Junpeng et al., 2007; Yang et al., 2008).

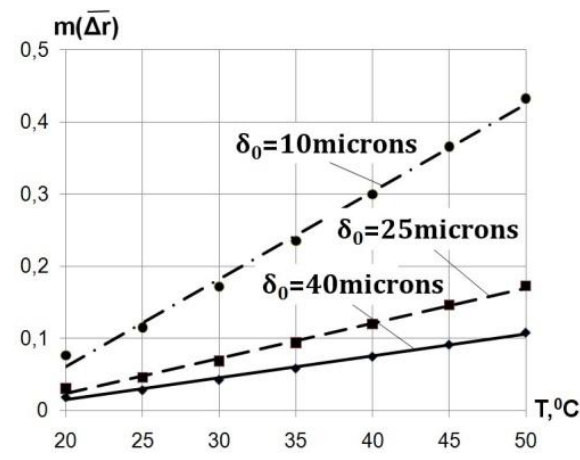

Fig. 3. Dependence of the mathematical expectation $m(\overline{\Delta r})$ value to temperature of support surface

Number $N, Q$ and harmonic $A_{k}^{b}, A_{k}^{S}$ amplitudes of HB profile support surfaces, were defined by spectral analysis of contour built by the results of numerical simulations of displacement radial coordinate profile support surfaces as a result of temperature (Fig. 4) and elastic deformation means by CAE-system SolidWorks Simulation (Yaun et al., 2008).

a)

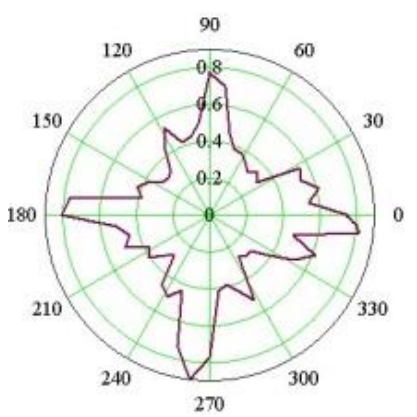

b)

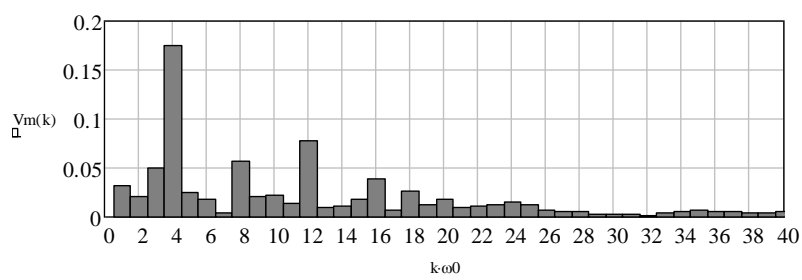

Fig. 4. Temperature displacement $\left(\mathrm{T}=35^{\circ} \mathrm{C}\right)$ of profile hydrostatic bearing surface of the bushing which approximated in package MathCAD by cubic splines a) and typical spectrum $b$ ) amplitudes $V_{m}(k)$ of harmonics profile $k^{*} \omega_{0}$

Taking into account all previous assumptions, simplifyings 
and calculations, the system of differential equations that describe the displacement of the spindle in the plane perpendicular to its geometrical axis, will look:

$\left\{\begin{array}{l}m \frac{d^{2} x}{d \tau^{2}}=\chi_{c} F_{C x}(\tau)-\chi_{F} R_{x}+\chi_{R} F_{p r x}(\tau)+\chi_{P} P_{x}(\tau), \\ m \frac{d^{2} y}{d \tau^{2}}=\chi_{c} F_{C y}(\tau)-\chi_{F} R_{y}+\chi_{R} F_{p r y}(\tau)+\chi_{P} P_{y}(\tau)+\chi_{G} m g,\end{array}\right.$

The components included in the right part of the equation system (17) are determined by the dependencies (6), (7), (11) - (15).

In general case, given the nonlinearity and the random nature of the components included in the right parts of equations, the system (17) has no analytical solution. This necessitates the use of numerical methods to solve it.

\section{THE RESULTS OF NUMERICAL SIMULATION TRAJECTORIES SPINDLE WITH HYDROSTATIC BEARINGS}

For numerical simulation of trajectories spindle is used statistical modeling by Monte Carlo (Rubinstein, 2007; Yu and Zhang, 2008). Description of spindle movements was carried out by means of radius vector in polar coordinates:

$R\left(\varphi_{j}\right)=\sqrt{\mathrm{x}^{2}\left(\tau_{j}\right)+y^{2}\left(\tau_{j}\right)}$

It was established that the workflow fluctuations in HB spindle is stationary and ergodic process that has a pronounced stochastic character in all ranges of variation of operating parameters and technological loads. Moreover the distribution law of a random variable spindle oscillations is close to normal. Therefore, the estimation of trajectories spindle parameter was performed using the statistical characteristics of the radius vector: $m_{R}$ mathematical expectation and standard deviation $\sigma_{R}$, which determine the displacement and dispersion of the spindle regulations and affect the accuracy of the nominal size of the treated surface.

According to the results of numerical simulations it was found that the increase of pressure in $\mathrm{HB}$ pockets from $1 \mathrm{MPa}$ to $3 \mathrm{MPa}$ enhances the precision machining that was shown in reducing the expectation of $m_{R}$ radius vector trajectories of the spindle with idealized form bearing surfaces - $30 \%$, with the errors of form bearing surfaces $-20 \%$. Mathematical expectation of the value of the radius vector spindle trajectories with ideal form of bearing surfaces in 1.2-1.4 times less compared to the spindle, HB of which have geometric deviation bearing surfaces (Fig. 5, b). The deviation form bearing surfaces of spindle in $\mathrm{HB}$ have little impact on the medium-deviation of radius vector trajectories (Fig. 5, b).

It was defined that for HB with idealized form of bearing surfaces expectation $m_{R}$ radius vector in the range $n=1500-2400 \mathrm{~min}^{-1}$ increases 1.85 times, while taking into account the forms errors of bearing surfaces in $\mathrm{HB} m_{R}$ increases in 1.4 times (Fig. 6,a). a)

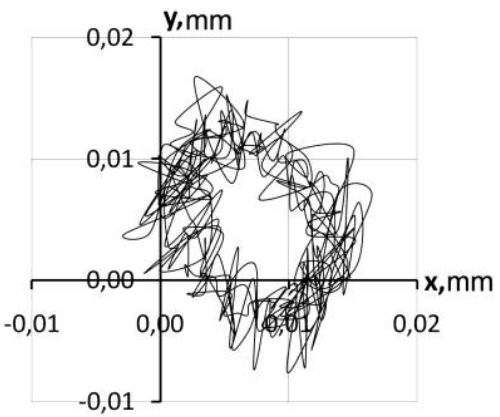

b)

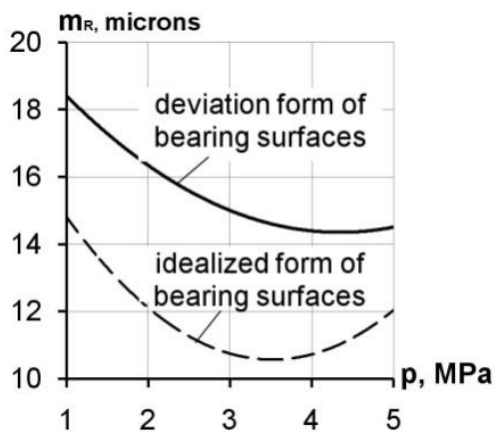

c)

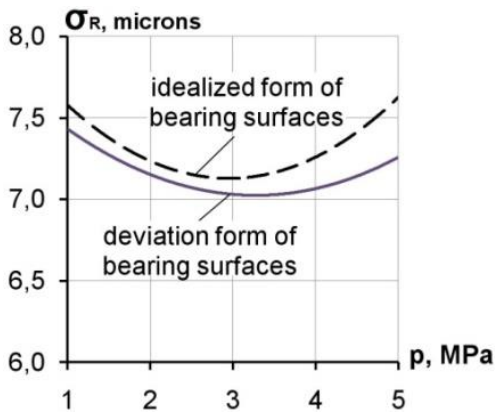

Fig. 5. Typical trajectory of 5 spindle turns: a) mathematical expectation; b) medium-square deviation; c) value of the radius vector trajectories in regulating of pockets pressure in $\mathrm{HB}$

$\left(n=1500 \mathrm{~min}^{-1}\right.$, Dst $\left.=4360 \cdot 10^{-6} \mathrm{~kg} \cdot \mathrm{m}\right)$

a)

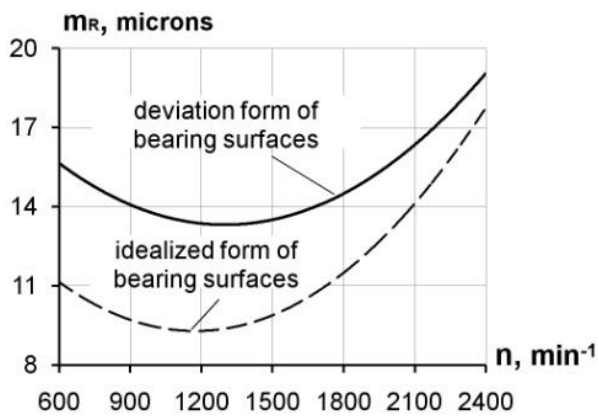

b)

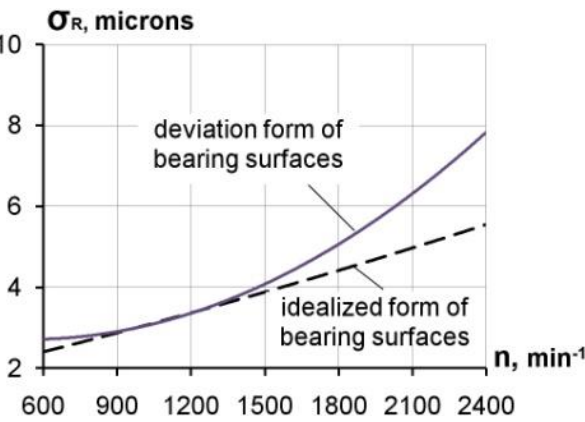

Fig. 6. Mathematical expectation a) and medium-square deviation b) of radius vector trajectories by varying frequency of spindle rotation 


\section{EXPERIMENTAL RESEARCH TRAJECTORIES OF THE SPINDLE WITH HYDROSTATIC BEARINGS}

Experimental studies were conducted to test the adequacy of the results obtained by numerical simulation. For this purpose, it was developed a set for experimental studies, implemented as a experimental stand based on precision lathe model UT16A (Sapon, 2013) (Fig. 7).

With increasing frequency of rotation the difference between the values of mathematical expectation $m_{R}$ of radius vector of trajectories spindle for HB with deviations and ideal form of bearing surfaces decreases from $40 \%$ for $n=600 \mathrm{~min}^{-1}$ to $8 \%$ for $n=2400 \mathrm{~min}^{-1}$. The deviation form bearing surfaces HB affects the value of standard deviation $\sigma_{R}$ of radius vector at frequency of rotation over $1500 \mathrm{~min}^{-1}$ (Fig. 6, b), at the same time with increasing frequency of rotation the influence of bearing surfaces forms on the precision of the spindle becomes more significant.

The tendency for increasing of the statistical characteristics of spindle precision with increasing rotation frequency is due to the influence of centrifugal force of inertia, which is proportional to the square of the angular velocity $\omega$ spindle. Reducing the value of $m_{R}$ in the range $n=600-1200 \mathrm{~min}^{-1}$ is due to the reduction of size cutting force with increasing cutting speed and constant $t_{n}$ and $S_{0}$.

As a result of spectral analysis of the frequency composition trajectories it is showed that the amplitude of harmonic spectra trajectories with deviation form of bearing surfaces in $\mathrm{HB}$ are at $15-60 \%$ more than harmonic trajectories with idealized form of bearing surface, confirming a significant impact on the accuracy of formative movements of spindle accuracy of the bearing surfaces.
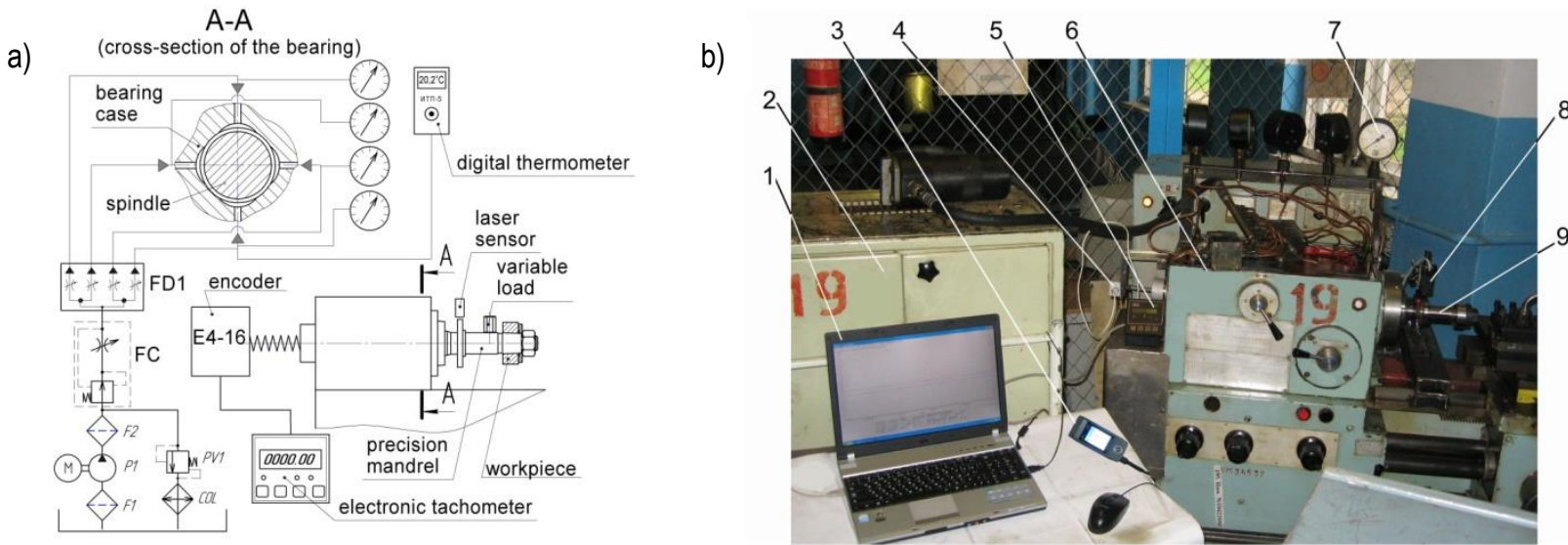

Fig. 7. Structural diagram (a) and general view (b) information-measuring complex for experimental studies of spindle accuracy with adjustable $H B$ : 1 - laptop; 2 - stations; 3 - thermometers; 4 - encoder; 5 - electronic tachometer; 6 - lathe UT16A; 7 - manometer; 8 -laser sensor; 9 - precision mandrel; FD1 - flow divider; FC - control flow valve; P1 - pump; F1, F2 - filters rough and fine cleaning; PV1 - relief valve; COL - oil chiller

The frequency of spindle rotation was fixed on the testimony of specialized electronic tachometer with accuracy of $\pm 0,05 \mathrm{~min}^{-1}$. The pressure in the HB pockets was defined by means of the flow regulator throttle type, and controlled pressure gauge with an accuracy of $0.1 \mathrm{MPa}$.

To determine the spindle displacements $\Delta \mathrm{x}$ and $\Delta \mathrm{y}$ in a plane perpendicular to the axis of rotation were used triangulation laser sensors model RF603.2-10/ 2-485-U-IN-AL-CG (Fig. 8, a), the working range of which is $2 \mathrm{~mm}$, the resolution is $-0.2 \mathrm{mkm}$, the maximum sampling frequency $f_{d}=9.4 \mathrm{kHz}$.

Two sensors were installed at $90^{\circ}$ on a rack 3 and in the measurements interacted with the control surface of precision mandrel 1 (Fig. 8, b), which was installed in a conical hole spindle machine.

The registration of signals from laser sensors were carried out by the analog-to-digital converter (ADC) based on a digital oscilloscope (Fig. 8, c). ADC connection with PC was carried out using usb interface.

The developed software on the basis of the Windows operating system provides registration, storage, conversion of input signals and then output to the display PC graphics as time dependencies in real time. To minimize the systematic error of the spindle rotation caused by deviations form the control surface of precision mandrels, fine finish control surface was carried out on this machine tool, and after processing mandrel was remained invariably fastened in the spindle for the entire series

of experimental studies. It made it possible to provide radial deviation of control surface within the limits of 1.0 microns.

a)
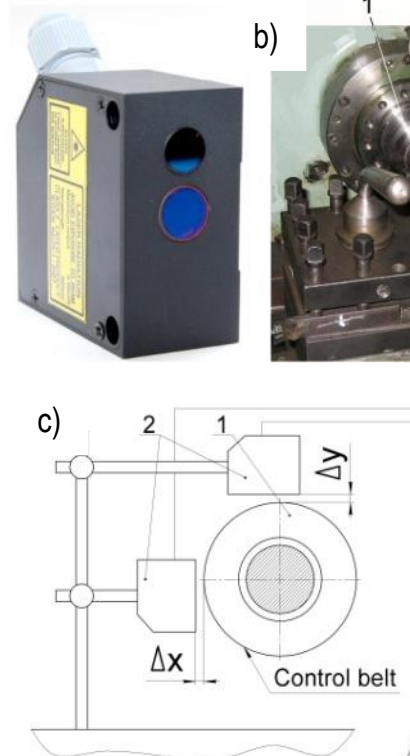

Fig. 8. Triangulation laser sensor a) general view b) schemes of fixing and block diagram measurement of spindle trajectories $\mathrm{c}$ ): 1 - precision mandrel; 2 - laser sensors; 3 - stand; 4 - stove 
To ensure the same number of measurements per rotation of the spindle during the experiments sampling frequency $A D C$ signal to change the proportion to the frequency spindle within $f_{d}=200-2500 \mathrm{~Hz}$ so as to ensure each sensor 50 measurements per rotation of the spindle. This accuracy allows to set statistical characteristics of the trajectories by varying operating parameters $\mathrm{HB}$ and discover new patterns in the test process.

Fig. 9 shows experimental trajectories of three spindle rotations, that are built on the results of measurements with sampling rates $f_{d}=200 \mathrm{~Hz}$ and $f_{d}=2000 \mathrm{~Hz}$ and static imbalance Dst $=$ $6525 \cdot 10^{-6} \mathrm{~kg} \cdot \mathrm{m}$. The resulting shape of the trajectories in Fig. 9 indicates the advisability of providing high sample rate ADC during the measurement.

It allowed to establish a discrepancy existing ideas about the ellipticity of the trajectory of the spindle on hydrostatic bearings terms of precision machining (Fig. 9). As shown in Fig.9, the trajectory of the spindle at HB have a pronounced stochastic character, indicating that the complicated nature of processes in lubricant layer of HB. This is explained by the influence of random errors of form, elastic and thermal strains bearing surfaces. The presence of the loop-shaped trajectories of movement is caused by the nonlinear properties of lubricating layer in resisting by the action unsteady external load.

a)

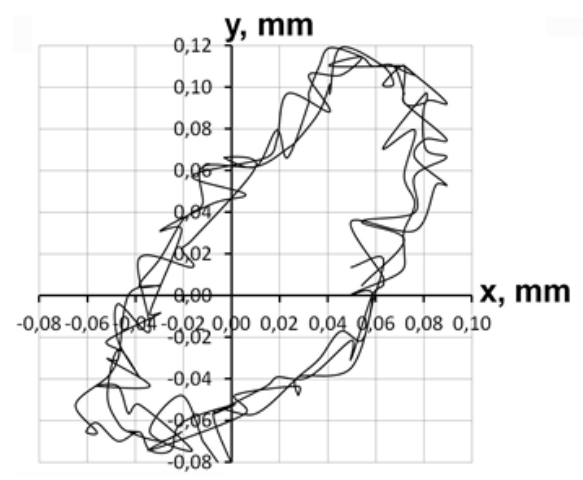

b)

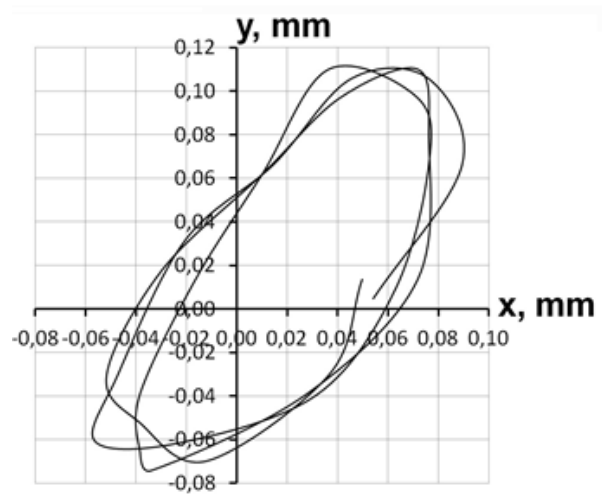

Fig. 9. The experimental trajectory of spindle lathe: $\mathrm{a}-f_{d}=2000 \mathrm{~Hz}$; $\mathrm{b}-f_{d}=200 \mathrm{~Hz}$

By the measurement results of experimental trajectories were defined their statistical value. The comparison of experimental and theoretical dependences of mathematical expectation and meansquare deviation of the radius vector of trajectories (Fig.10 a, b) and frequency spindle rotation (Fig. $10 \mathrm{c}$, d) shows their qualitative consistency.

The maximum difference between the experimental and theoretical values of mathematical expectation of a radius vector, regarding deviations form of bearing surfaces does not exceed
$16.7 \%$ with regulating pressure in the pockets and $18.8 \%-$ by varying the rotation speed of the spindle. Compared with the values of mathematical expectation radius vector trajectories obtained by mathematical model with an idealized bearing surface, experimental values differ on average in 1.5 times, it shows the importance of taking into account the forms of bearing surfaces in the study of HB precision spindle.

a)

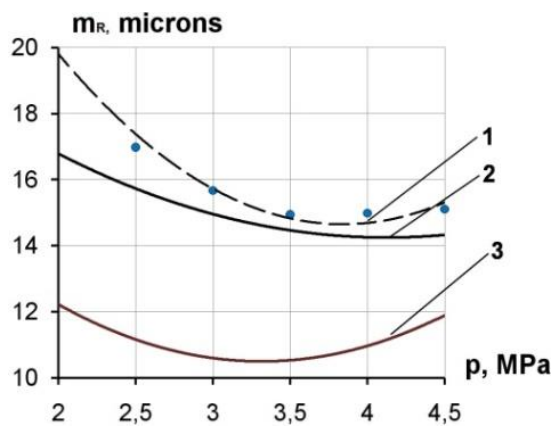

b)

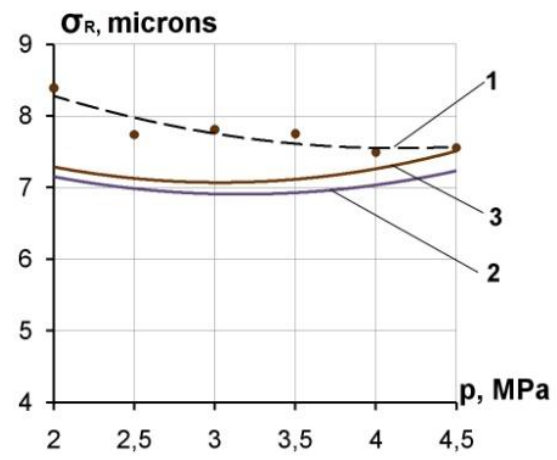

c)

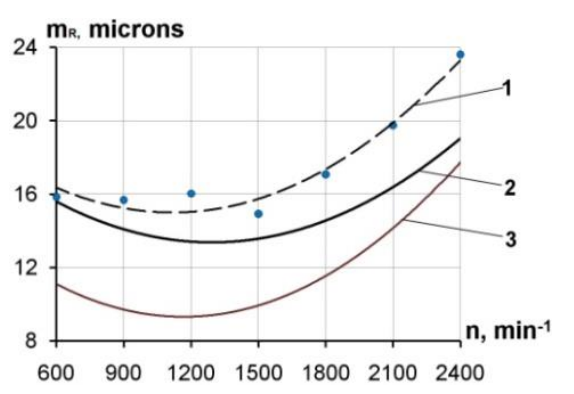

d)

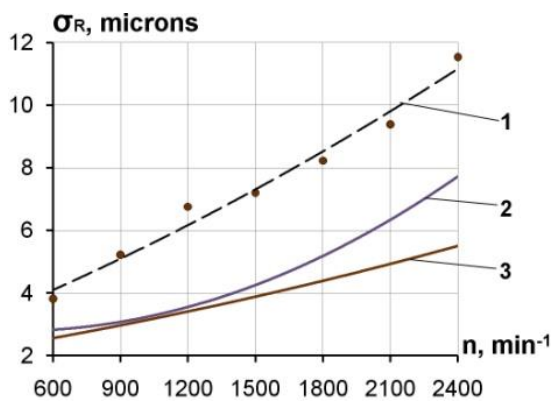

Fig. 10. Comparison of experimental and theoretical dependences of mathematical expectation and mean-square deviation of the radius vector of trajectories a), b) at $\mathrm{n}=1500 \mathrm{~min}^{-1}$ and the rotational speed of the spindle c) and d) at $\mathrm{p}=3 \mathrm{MPa}$ : 1 - experimental dependence; 2 - theoretical dependence considering deviations form the supporting surfaces;

3 - theoretical dependence with idealized bearing surface

The maximum difference between the experimental and theoretical values mean-square deviation of the radius vector trajectories obtained taking into account the deviation form bearing sur- 
faces does not exceed $14.3 \%$ - with regulating pressure in pockets of $\mathrm{HB}$ and $43 \%$ - by varying the rotation speed of the spindle. The difference between the experimental and theoretical values mean-square deviation of spindle trajectories by varying the rotation speed of the spindle is due to the influence of centrifugal force of inertia caused by the presence of static imbalance, on vibration spindle unit which parameters are quite difficult to describe analytically in a mathematical model.

\section{CONSTRUCTION OF ADJUSTABLE HYDROSTATIC BEARING}

One of the most perspective directions to improve the accuracy of machine tools is to develop new and upgrade existing structures spindle units by applying in them hydrostatic bearings adjustable type.

Adjusting the operating and design parameters of hydrostatic bearings allows to expand technological capabilities of machines, increase processing performance, reduce operating costs, allows accurate control of formative movement tools, from which to perform the accuracy of the size, shape and relative position of parts of machined surfaces are dependent.

In Chernihiv National University of Technology a new design adjustable hydrostatic bearing has been developed (Fig. 11).
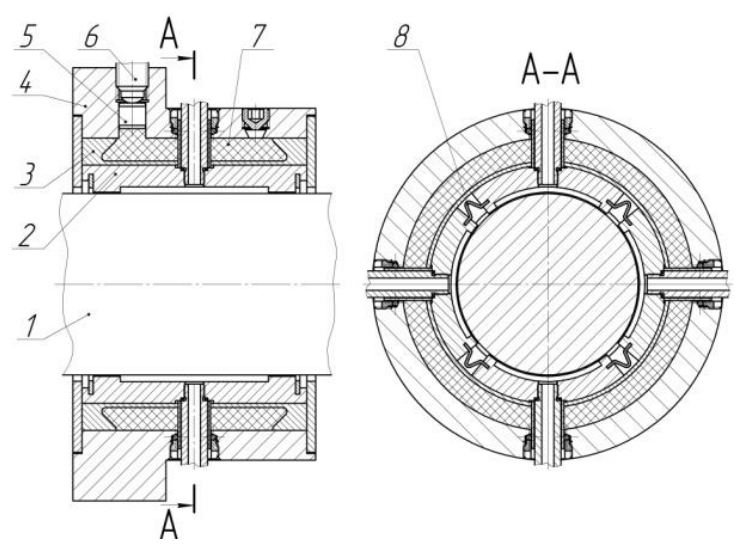

Fig. 11. Adjustable hydrostatic bearing 1 - spindle; 2 - segment; 3 - elastic sleeve; 4 -housing; 5 - plunger; 6 - screw; 7 - hydro-plastic; 8 - springs

In the basic design of the adjustable hydrostatic bearing was assigned the task of improving the uniformity of the adjustment radial clearance, maintenance of repair capability and technological design of the bearing.

The adjusting of the radial clearance in the bearing is made by turning the screw 6 , spherical end face of which presses the plunger 5 , creating hydrostatic pressure in the cavity filled with hydro-plastic 7.Under the influence of hydrostatic pressure of hydro-plastic theuniform deformation of a thin-walled sleeve 3 , takes place resulting in 2 segments movement radially decreasing radial clearance between the bearing surface of the spindle 1 and segments. The deflection of segments is regulated by hydrostatic pressure that is created in the cavity with hydro-plastic.

The benefits of the design: the surface of the hydrostatic bearing is made of 4 individual segments 2 , connected by springs 8 of special shape and the regulation of radial clearance in the bearing is provided by simultaneous radial displacement of 4 segments due to uniform deformation of elastic thin-walled sleeve
3 under hydro-plastic pressure.

Built-up construction of hydrostatic bearing surface, connected by springs of special shape allows to increase adaptability, ability to repair bearing and expand the range of structural materials for the manufacture of the bearing. The minimum anisotropy of physical and mechanical properties, precision of shapes and sizes of springs 8 of special shape is achieved by using a calibrated rolled metal sheets as the original piece and finish shaping pressure. Named design and technological options will provide uniform radial displacement of segments 2 , which will increase to 1.3-1.5 times control the amount of precision radial clearance in the bearing.

\section{CONCLUSIONS}

It was developed the mathematical model ofspindle trajectories on hydrostatic bearings, which takes into account the influence of design parameters, geometrical deviations form deformation temperature bearing surfaces of the bearing, random and deterministic operating parameters hydrostatic bearings and technological loads, it allows to increase by $30-60 \%$ the accuracy of the spindle position.

It was defined that accounting errors of shape in transverse direction and temperature strain bearing surfaces of the hydrostatic bearing in modeling trajectories spindle leads to an increase in the statistical characteristics of the radius vector paths: the mathematical expectation - by $30 \%$, mean-square deviation - $12.5 \%$ compared to idealized bearing surface of the bearing.

As a result of numerical modeling was defined and statistical patterns of precision of the lathe by terms of precision spindle movement trajectories in hydrostatic bearings, the results were verified experimentally:

- increasing the rotational speed of the spindle in the range of $600-2400 \mathrm{~min}^{-1}$ in the presence of static imbalance of the spindle unit causes increase in 1.6-2.1 times the mathematical expectation and 2.5-3.4 times the mean-square deviation radius-vector of trajectories spindle;

- rational value of hydrostatic pressure in bearing pockets in which the minimum value provided by the mathematical expectation and mean-square deviation of the radius vector trajectories is in the range of 3-3.5 MPa.

It was developed a new design of adjustable hydrostatic bearing, which can increase to 1.3-1.5 times the precision control of the radial clearance, bearing manufacturing technology and expand the range of structural materials for the manufacture of bearing elements.

\section{REFERENCES}

1. Fedorynenko D., Boyko S., Sapon S. (2015), The search of the spatial functions of pressure in adjustable hydrostatic radial bearing, Acta Mechanica et Automatica, 9(1), 23-26.

2. Fedorynenko D., Sapon S., Boyko S.(2014), Considering of the thermal strains in determining the function of the radial clearance in hydrostatic bearing in high-speed spindle node, Technological systems, 2(10), 154-159.

3. Fedorynenko D., Sapon S., Boyko S., Kosmach A. (2015), Information-measuring complex for research of spindle trajectories on hydrostatic bearings, Scientific Bulletin of National Mining University: scientific journal, 6(150), 42-48. 
4. Junpeng S., Guihua H., Yanqin Z., Yuhong D. (2008), Hardware-inthe-loop Simulation on Controllable Hydrostatic Thrust Bearing, IEEE International Conference on Automation and Logistics (ICAL 2008), 1095-1099.

5. Junpeng S., Yanqin Z., PengchengL. (2007), Static flow simulation of hydrostatic bearing ellipse and sector curve based on fluent, Lubrication Engineering, 1, 93-95.

6. Perovic B. (2012), Hydrostatic guides and bearings: basic principles, calculation and design of hydraulic plans (in German), SpringerVerlag Berlin Heidelberg.

7. Rubinstein R.Y. (2007), Simulation and the Monte Carlo Method, Kroese - 2nd edition, Wiley.

8. Sapon S.P.(2013), Methodology of experimental determination precisionspindle, Bulletin of Chernihiv State Technological University, A series of technical sciences, 1(63), 66-74.

9. Savin L.A. (2006), Simulation of rotor systems with fluid friction bearings, Moscow: Engineering.

10. Shen C.G., Wang G.C., Wang S.L. (2010), Computation and Analysis of Unbalancing Responses of High Speed Machining Tool System, Advanced Materials Research, 148-149(1), 40-46.

11. Solomin O.V.(2007), Development of methods and tools of dynamic analysis of rotor systems with fluid friction bearings, phd thesis, Orel State University.

12. Strutynsky V., Fedorynenko D. (2011), Statistical dynamics of spindle units for hydrostatic bearings, Nizhin: LLC "Publishing" Aspect-Polygraph.

13. Wardle F. (2015), Ultra Precision Bearings, Cambridge: Elsevier.
14. Xiaodong Y., Huaimin L. (2006), Computerized Simulation of Lubricating Characteristics of Circular Tilting Pad Thrust Bearing, Lubrication Engineering, 3, 84-87.

15. Xiaodong Y., Huaimin L., Xiurong G. (2007), Numerical Analysis of Lubricating Characteristics of Sector Thrust Bearing Pad, Lubrication Engineering, 1, 123-125.

16. Yu X.D., Zhang Y.Q. (2008), Numerical Simulation of Gap Flow of Sector Recess Multi-pad Hydrostatic Thrust Bearing, Proc. 2008 Asia Simulation Conference-7th Int. Conf. Simulation and Scientific Computing (ICSC 08), 675-679.

17. Yuan S., Lin J., Liu Q. (2008), Finite Element Analysis of Machine Tool as a Whole, Machine Tool \& Hydraulics,36(4):17-18,49.

18. Zhao H., Yang J., Shen J.(2007), Simulation of thermal behaviour of a CNC machine tool spindle, International Journal of Machine Tool \& Manufacture, 47(6), 1003-1010.

Acknowlegement: The presented results are part of projects 0113 U000503 "Development of the Precision-Controlled Hydraulic Bearings of High-Speed Spindle Units" and 0115 U002362 "Development of Energy-Efficient High-Speed Spindles with the Adaptive Journal Bearings" funded by the Government of Ukraine. 